\title{
Distribución en planta del laboratorio de pintura de la Escuela Naval de Suboficiales ARC "Barranquilla"
}

\author{
Cindy Paola Charris Maldonado \\ Centro de Investigaciones, Escuela Naval de Suboficiales A.R.C. "Barranquilla”, Barranquilla, Colombia. \\ cindycharris85@gmail.com
}

Recibido: Marzo 28, 2020.

Recibido en su versión corregida: Abril 17, 2020.

Aceptación: Abril 24, 2020.

Cómo citar: Charris Maldonado, C.P. (2020). Distribución en planta del laboratorio de pintura de la Escuela Naval de Suboficiales ARC "Barranquilla". Revista Sextante, 22, pp. 1 - 8, 2020.

\section{Resumen}

En el presente proyecto se realizó la propuesta de una óptima distribución para el laboratorio de materiales compuestos de la Escuela Naval de Suboficiales ARC "Barranquilla" (ENSB), con el propósito de mejorar el proceso de producción de pintura vinilo tipo I elaborado en la institución.

Para obtener los resultados esperados el proyecto se desarrolló en tres fases. En la fase 1, a través del uso de herramientas como el análisis de factores se realizó un análisis de la situación actual del proceso de producción de pintura y la distribución del laboratorio, con los resultados obtenidos se desarrollaron los diagramas asociados a la elaboración de la pintura, se identificaron las falencias y se propusieron algunas mejoras dentro del proceso; en la fase 2, con ayuda del software IIE Microcraft, se estableció un diseño de redistribución que busca optimizar tiempo, espacios y costos derivados del proceso de producción de pintura; y por último se efectuó un análisis comparativo de los costos generados por la distribución actual y los costos generados por la distribución propuesta, con los resultados obtenidos se redujo el riesgo de enfermedades profesionales y de accidentes de trabajo, eliminando lugares inseguros, pasos peligrosos y materiales de pasillos. Se obtuvo un menor número de retrasos, reduciéndose los tiempos de espera, al equilibrar los tiempos de trabajo y se disminuyeron las distancias de recorrido.

Palabras claves: Distribución; Estudio de métodos; Estudio de tiempos; Fabricación; Optimización; Procesos.

\section{Layout of the Painting Laboratory of the Escuela Naval de Suboficiales ARC "Barranquilla"}

\begin{abstract}
In this project, the proposal was proposed for an optimal distribution for the composites laboratory of the ARC "Barranquilla" Naval School of Officers (ENSB), with the aim of improving the production process of type I vinyl paint developed at the institution.

To obtain the expected results, the project was developed in three phases, in phase 1 through the use of tools such as factor analysis and studies of methods and times an analysis was carried out of the current situation of the paint production process and the distribution of the laboratory, with the results obtained the diagrams associated with the elaboration of the painting were made, the gaps were identified and some improvements were proposed within the process; in Phase 2 with the help of the IIE Microcraft software, a redistribution design is established that seeks to optimize time, spaces and costs arising from the paint production process and finally a comparative analysis was carried out of the costs generated by the current distribution and the costs generated by theproposed distribution, with the results obtained reduced the risk of occupational diseases and work accidents, eliminating unsafe places, dangerous steps and materials from corridors. Fewer delays were achieved, reducing waiting times, balancing working times and decreasing travel distances.
\end{abstract}

Keywords: Distribution; Manufacturing; Method study; Optimization; Processes; Time study. 


\section{Introducción}

"La distribución en planta está asociada con la disposición de las máquinas, las estaciones de trabajo, los departamentos, las zonas de almacenamiento, los corredores y las áreas en común dentro del establecimiento productivo propuesto o ya existente. La finalidad primordial de la distribución en planta radica en estructurar estos elementos de manera que se garantice la fluidez del flujo de trabajo, materiales, personas e información por medio del sistema productivo" (López, 2017). También, se quiere hallar con esto una organización de las áreas de trabajo y equipo, de forma más económica, avalando la seguridad y satisfacción de los empleados.

La Escuela Naval de Suboficiales ARC "Barranquilla", dentro de sus instalaciones cuenta con el laboratorio de materiales compuestos, donde se lleva a cabo el proceso de producción de pintura vinilo tipo I; este no posee la distribución adecuada, dificultando la movilidad del operario, ocasionando desperdicios de material, pérdida de tiempo y recorridos más largos.

Con el desarrollo de este proyecto se logró determinar cuál es la propuesta de la distribución de planta del laboratorio de materiales de la ENSB, que permita optimizar la disposición de elementos en el área de producción, enfocándose en factores como máquinas, recursos humanos y materiales, haciendo que la propuesta realizada incremente los niveles de eficiencia del proceso de producción de pintura y sea una alternativa para su uso en la infraestructura de la institución.

Los impactos que pueden producirse a través de la implementación de esta propuesta son: la estandarización del proceso, optimización de los recursos y una nueva distribución del área de trabajo.

\section{Metodología}

La metodología propuesta para el desarrollo de este proyecto se divide en 3 fases cada una de ellas con diferentes actividades que ayudarán al cumplimiento del objetivo. (ver Figura 1)

\section{Desarrollo}

\subsection{Diagnóstico de la situación actual del proceso y de la distribución del laboratorio}

El laboratorio de materiales compuestos de la ENSB cuenta con un área total de $39 \mathrm{~m} 2$, a su vez se divide en dos secciones, la primera tiene dimensiones de 6 metros de largo y 5 metros de ancho, para poder tener mejor control de esta área se decidió dividirla en tres departamentos, en los que se realizan diferentes actividades relacionadas con el proceso de producción de pintura vinilo tipo I.

La segunda sección es más pequeña, con dimensiones de 3 metros de largo y 3,30 metros de ancho, está dividida en tres departamentos.

De acuerdo con lo investigado en los textos de distribución en planta y análisis de la producción de las operaciones, se distinguen 4 tipos de distribución en planta: posición fija, por procesos, por producto y por células o hibridas (Muther, 1970); (Nahmias, 2007). El tipo de distribución utilizada en la elaboración de pinturas es por proceso, ya que el material va a través de diferentes departamentos antes de llegar a la maquina homogenizadora, pero el producto no es elaborado de manera continua, solo se fabrica por pedido solicitado, es decir producción intermitente, este tipo de distribución agrupa máquinas que tienen funciones parecidas. Las distribuciones de procesos son muy efectivas cuando hay una amplia variación en la mezcla del producto (Nahmias, 2007).

En la Figura 2 se muestran los planos de la distribución actual del laboratorio de materiales compuestos en donde se lleva a cabo el proceso de producción de pintura vinilo tipo I.

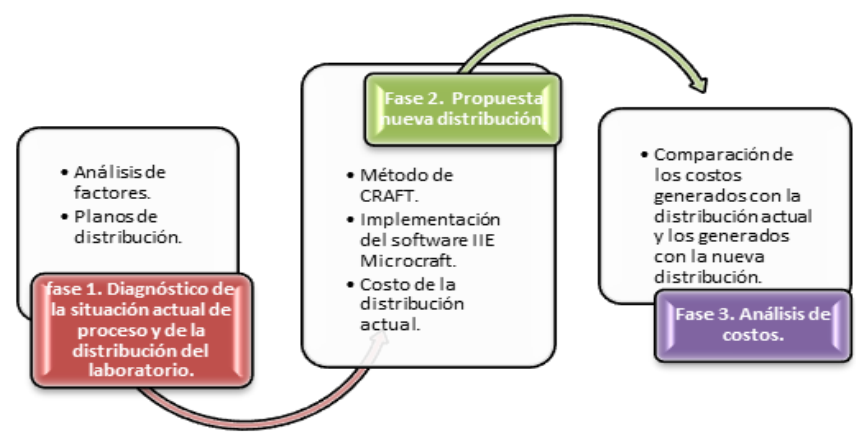

Figura 1. Metodología desarrollada.

Fuente: Los autores. 


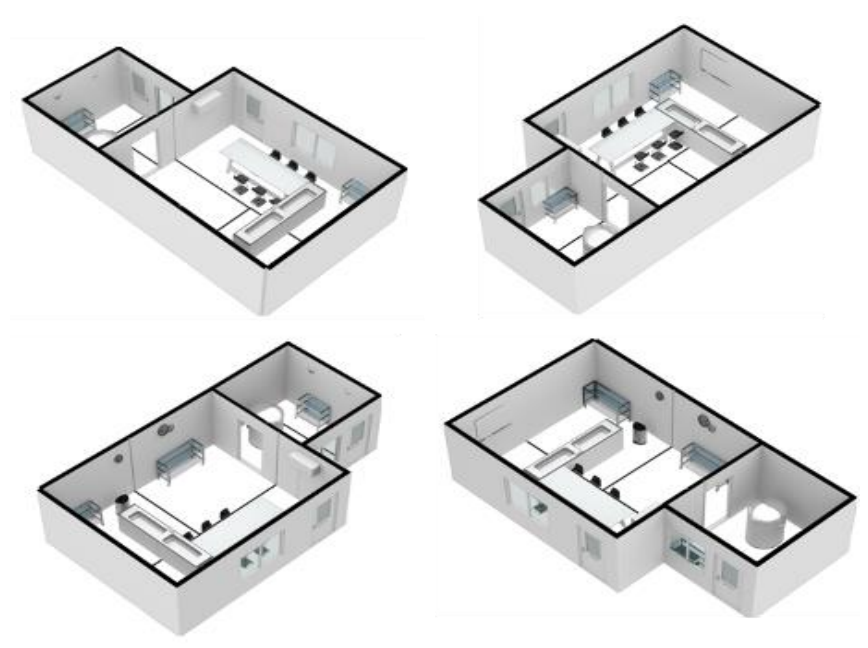

Figura 2. Distribución actual laboratorio de materiales compuestos ENSB.

Fuente: Los autores.

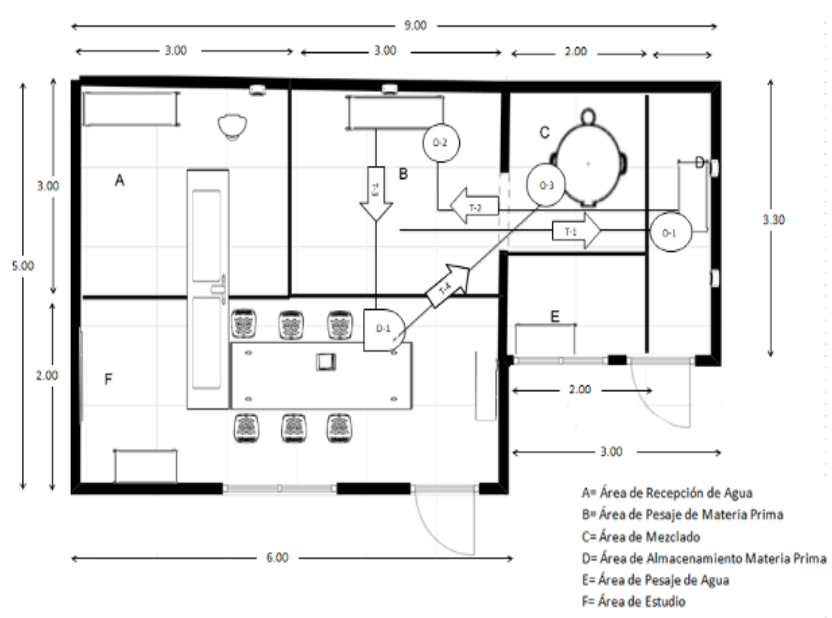

Figura 3. Diagrama de recorrido actual (metros).

Fuente: Los autores.

Actualmente el proceso de producción de pintura vinilo tipo I que se realiza en la ENSB, no cuenta con diagramas que permitan controlar el proceso, por tal motivo se decide realizar observaciones, con el fin de obtener información pertinente de cada una de las actividades que se llevan a cabo para producir pintura tipo I, la cual es necesaria en el momento de determinar los costos de fabricación de dicho producto.

También nos ayuda a determinar falencias en los actuales métodos utilizados para la producción y así poder proponer acciones de mejora. (ver Figura 3)

Para identificar las falencias dentro del procesos de producción de pintura vinilo tipo I, se realizó un análisis de factores. Estos factores están divididos en 8 grupos: material, maquinaria, hombre, movimiento, espera, servicios, edificio y cambio (Platas \& Cervantes, 2014).
Los resultados obtenidos del análisis de los factores se muestran en la Figura 4.

\subsection{Propuesta nueva distribución}

Para la elaboración de la nueva propuesta de distribución del laboratorio de materiales compuestos de le ENSB se utilizó el método de CRAFT. Este es un programa empleado para mejorar distribuciones. Puede operar hasta 40 departamentos.

$\mathrm{Su}$ sigla CRAFT significa Computerized Relative Allocation of Facilities o Asignación Relativa Computarizada de Instalaciones. Tiene como finalidad minimizar el costo total de transporte de una distribución. Coronado en su blog Jaracohe nos muestra un informe de métodos para distribución en planta donde explica que "El costo de transporte es el resultado de la suma de todos los elementos de una matriz de flujos (matriz desde hacia cada departamento), multiplicado por la distancia y el costo por unidad de distancia recorrida de un departamento a otro. La función del costo de transporte puede cambiarse por cualquier otra función que represente el costo de una "relación" entre cualquier par de departamentos" (Coronado \& Mejía, 2007).

Para la implementación del método CRAFT se realizó la distribución de cada uno de los departamentos que intervienen en el proceso de producción de pintura vinilo tipo I. (ver Tabla 1).

Teniendo en cuenta cada uno de los recorridos que realiza la materia prima, se elaboraron las diferentes matrices (flujos, costos y distancia) que nos ayudaron a determinar el costo de la distribución actual. Cabe aclarar que en la ENSB se realiza pintura vinilo durante tres días, cada seis meses, es decir, seis días al año. El proceso de fabricación para 25 Galones tiene una duración de cuatro horas, lo que quiere decir que el operario en una jornada laboral realiza dos veces pintura (Lo que indica que al año se realiza doce veces este procedimiento) obteniendo un total de 50 galones. Al término de los tres días se producen 150 galones, de modo que anualmente se elaboran 300 galones.

La matriz de flujos anuales representa la cantidad de veces que el operario se desplaza de un departamento a otro durante el proceso de fabrica- 


\begin{tabular}{|c|c|c|c|c|c|}
\hline $\begin{array}{l}\text { Factor material } \\
\text { Desperdicio de } \\
\text { material. } \\
\text { Derrame de } \\
\text { pintura al envasar } \\
\text { el producto final. }\end{array}$ & 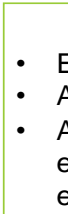 & $\begin{array}{l}\text { tor servicio } \\
\text { gía: } 220 \text { V. } \\
\text { : Bueno } \\
\text { tarillado: se } \\
\text { entra en mal } \\
\text { to. }\end{array}$ & $\begin{array}{l}\text { Factor es } \\
\text { Existen esperas } \\
\text { proceso de prep } \\
\text { de MP antes de } \\
\text { agregadas a la } \\
\text { mezcladora. }\end{array}$ & $\begin{array}{l}\text { ra } \\
\text { el } \\
\text { ación } \\
\text { quina }\end{array}$ & $\begin{array}{l}\text { Factor movir } \\
\text { Se realizan reco } \\
\text { largos por la } \\
\text { inadecuada dist } \\
\text { de espacios en } \\
\text { laboratorio. }\end{array}$ \\
\hline $\begin{array}{l}\text { Factor maquin } \\
\text { - Se utilizan botellas } \\
\text { plásticas reciclada } \\
\text { recipientes para m } \\
\text { MP. } \\
\text { - Una de las balanza } \\
\text { digitales a usar est } \\
\text { deteriorada. }\end{array}$ & $\begin{array}{l}\text { ia } \\
\text { como } \\
\text { lir la } \\
\text { muy }\end{array}$ & $\begin{array}{l}\quad \text { Fac } \\
\text { Se analizan la } \\
\text { trabajo } \\
\text { - } \quad \text { Polvo: Abu } \\
\text { - } \text { Olor: Fuer } \\
\text { - } \text { lluminació } \\
\text { - Elementos } \\
\quad \text { Existen pe }\end{array}$ & $\begin{array}{l}\text { lombre } \\
\text { diciones de } \\
\text { a } \\
\text { son utilizados. }\end{array}$ & $\begin{array}{l}\mathbf{F} \\
\text { - Su } \\
\text { en } \\
\text { - } \mathrm{Pa} \\
\text { es } \\
\text { - } \mathrm{Ve} \\
\text { co } \\
\text { air }\end{array}$ & $\begin{array}{l}\text { or Edificio } \\
\text { : antideslizantes } \\
\text { al estado. } \\
\text { les: Buen } \\
\text { o } \\
\text { ación: cuenta } \\
\text { extractores y } 1 \\
\text { condicionado. }\end{array}$ \\
\hline
\end{tabular}

Figura 4. Matriz de flujos anuales (en metros).

Fuente: Los autores.

-ción de pintura. Para realizar la siguiente matriz anual, se multiplicó la cantidad de desplazamientos realizados durante el proceso, por doce (cantidad de veces que se fabrica pintura al año), teniendo como resultado un total de 1.356 desplazamientos anuales descritos en la Tabla 2.

La matriz de costos por viajes (ver Tabla 3 ) nos muestra el costo (en pesos) que tiene realizar los recorridos de un departamento a otro. Para elaborar esta matriz se debe tener en cuenta el costo de cada desplazamiento, el cual se obtuvo hallando el salario diario del operario, multiplicado por 6 , la cantidad de días al año que se realiza pintura, dándonos como resultado $\$ 733.800$, que vendría siendo el salario anual, luego se dividió este valor entre el total de recorridos anuales obteniendo un costo de $\$ 541$, lo que corresponde al valor de cada desplazamiento. Después se multiplica el recorrido anual entre departamentos por el valor del desplazamiento.

La matriz de distancias recorridas (ver Tabla 4) nos permite saber la distancia que hay entre departamentos. Para realizar esta matriz fue necesario hallar los centroides de cada departamento, luego se procedió a contar las distancias existentes entre el centroide de un departamento y otro.

Finalmente se determinó el costo total de la distribución actual (ver Tabla 5), multiplicando el costo por viajes anual de cada departamento por la distancia recorrida entre departamentos. Una vez se tiene el costo por departamento se suman todos los valores, obteniendo como resultado $\$ 3.074 .817$ que corresponden al costo total anual de la distribución actual del Laboratorio de Materiales Compuestos de la ENSB.
Tabla 1. Distribución del laboratorio de materiales compuestos en departamentos.

\begin{tabular}{cc}
\hline A & Agua \\
\hline B & Zona de pesaje de materia \\
C & prima \\
D & Aroceso de mezclado \\
E & Pesaje de agua \\
F & Área de estudio \\
\hline
\end{tabular}

Fuente: Los autores.

Tabla 2. Matriz de flujos anuales (en metros).

\begin{tabular}{ccccccc}
\hline & A & B & C & D & E & F \\
\hline A & & 12 & 36 & 0 & 60 & 0 \\
B & 12 & & 144 & 132 & 0 & 132 \\
$\mathbf{C}$ & 36 & 144 & & 0 & 0 & 132 \\
$\mathbf{D}$ & 0 & 132 & 0 & & 0 & 0 \\
$\mathbf{E}$ & 60 & 0 & 60 & 0 & & 0 \\
$\mathbf{F}$ & 0 & 132 & 132 & 0 & 0 & \\
\hline Total & & & & & & $\mathbf{1 3 6 5}$ \\
\hline
\end{tabular}

Fuente: Los autores.

Tabla 3. Matriz de costos por viajes (en pesos).

\begin{tabular}{ccccccc}
\hline & A & B & C & D & E & F \\
\hline A & 0 & 6494 & 19481 & 0 & 32469 & 0 \\
B & 6494 & 0 & 77926 & 71432 & 0 & 71432 \\
C & 19481 & 77926 & 0 & 0 & 0 & 71432 \\
D & 0 & 71432 & 0 & 0 & 0 & 0 \\
E & 32469 & 0 & 32469 & 0 & 0 & 0 \\
F & 0 & 71432 & 71432 & 0 & 0 & 0 \\
\hline
\end{tabular}

Fuente: Los autores.

Tabla 4. Matriz de distancias recorridas (en metros).

\begin{tabular}{ccccccc}
\hline & $\mathbf{A}$ & $\mathbf{B}$ & $\mathbf{C}$ & $\mathbf{D}$ & $\mathbf{E}$ & $\mathbf{F}$ \\
\hline & & & & & & \\
$\mathbf{A}$ & & 3 & 5,5 & 7 & 7 & 3,5 \\
$\mathbf{B}$ & 3 & & 2,5 & 4 & 3 & 3,5 \\
$\mathbf{C}$ & 5,5 & 2,5 & & 1,5 & 1,5 & 6 \\
$\mathbf{D}$ & 7 & 4 & 1,5 & & 2,5 & 7,5 \\
$\mathbf{E}$ & 7 & 3 & 1,5 & 2,5 & & 6,5 \\
$\mathbf{F}$ & 3,5 & 3,5 & 6 & 7,5 & 6,5 & \\
\hline
\end{tabular}

Fuente: Los autores. 
Para obtener la distribución propuesta a través del método heurístico CRAFT, se hace uso del programa Excel. Una vez que definimos el costo de la distribución actual, determinamos las posibles interacciones teniendo en cuenta que el departamento A y $\mathrm{F}$ no pueden ser reubicados, para esto utilizamos el software IIE-Micro-Craft, dando como resultado tres posibles interacciones que disminuyen el costo de la distribución actual:

- Interacción D-E

- Interacción C-B

- Interacción E-B

Para cada uno de los intercambios se muestran en la Tabla 6 , la Tabla 7 y la Tabla 8.

Teniendo en cuenta los resultados de las interacciones se obtuvo que la alternativa C-B disminuyó el costo de la distribución actual; a continuación, en la Figura 5 se muestra la nueva distribución.

Después de redistribuir los departamentos se calculan sus centroides y se determinan las nuevas distancias, tal como se muestra en la Tabla 9, la Tabla 10 y la Tabla 11.

Con base a los resultados obtenidos se determina el costo de la nueva distribución. Se observa que al realizar la nueva distribución su costo disminuye a $\$ 2.886 .496$. Realizamos nuevamente las posibles interacciones B-E, B-D y D-E.

En la Tabla 12, Tabla 13 y Tabla 14 se relacionan los resultados de intercambios a partir de la nueva distribución que no presenta mejoría. Con esta interacción se obtuvo una nueva distribución que disminuye el costo actual. En la Figura 6 se muestra la nueva distribución. Las posibles interacciones para esta nueva distribución son: B-E y B-D.

Al analizar los resultados de estas nuevas interacciones se observó que no mejora, así que se detiene el algoritmo. Teniendo en cuenta cada uno de los costos obtenidos en las diferentes interacciones se obtuvo que la distribución que disminuye el costo actual es C-B \$2.636.485. En la Figura 7 y Figura 8 se presentan las nuevas distribuciones físicas por método de CRAFT implementado.
Tabla 5. Matriz costo total de la distribución actual (en pesos).

\begin{tabular}{ccccccc}
\hline & A & B & C & D & E & F \\
\hline A & 0 & 19481 & 107148 & 0 & 227283 & 0 \\
B & 19481 & 0 & 194814 & 285727 & 0 & 250012 \\
C & 107148 & 194815 & 0 & 0 & 0 & 428591 \\
D & 0 & 285727 & 0 & 0 & 0 & 0 \\
E & 227283 & 0 & 48704 & 0 & 0 & 0 \\
F & 0 & 250012 & 428591 & 0 & 0 & 0 \\
\hline \multicolumn{7}{r}{ Total } \\
\hline
\end{tabular}

Fuente: Los autores.

Tabla 6. Matriz de intercambios D-E (en pesos).

\begin{tabular}{ccccccc}
\hline & A & B & C & D & E & F \\
\hline A & 0 & 19481 & 107148 & 0 & 227283 & 0 \\
B & 19481 & 0 & 194814 & 214296 & 0 & 250012 \\
C & 107148 & 194814 & 0 & 0 & 0 & 428591 \\
D & 0 & 214296 & 0 & 0 & 0 & 0 \\
E & 227283 & 0 & 48704 & 0 & 0 & 0 \\
F & 0 & 250012 & 428591 & 0 & 0 & 0 \\
\hline \multicolumn{7}{c}{ Total } \\
\hline
\end{tabular}

Fuente: Los autores.

Tabla 7. Matriz de intercambios C-B (en pesos).

\begin{tabular}{rrrrrrr}
\hline & A & B & C & D & E & F \\
\hline A & 0 & 35716 & 58444 & 0 & 194814 & 0 \\
B & 35716 & 0 & 194814 & 107148 & 0 & 428591 \\
C & 58444 & 194814 & 0 & 0 & 0 & 250012 \\
D & 0 & 107148 & 0 & 0 & 0 & 0 \\
E & 194814 & 0 & 97407 & 0 & 0 & 0 \\
F & 0 & 428591 & 250012 & 0 & 0 & 0 \\
\hline \multicolumn{7}{c}{ Total } \\
\hline
\end{tabular}

Fuente: Los autores.

Tabla 8. Matriz de intercambios E-B (en pesos).

\begin{tabular}{rrrrrrr}
\hline & A & B & C & D & E & F \\
\hline A & 0 & 38963 & 107148 & 0 & 97407 & 0 \\
B & 38963 & 0 & 116888 & 178580 & 0 & 464307 \\
C & 107148 & 116888 & 0 & 0 & 0 & 428591 \\
D & 0 & 178580 & 0 & 0 & 0 & 0 \\
E & 97407 & 0 & 81173 & 0 & 0 & 0 \\
F & 0 & 464307 & 428591 & 0 & 0 & 0 \\
\hline \multicolumn{7}{c}{ Total } \\
\hline
\end{tabular}

Fuente: Los autores.

Tabla 9. Nuevos centroides (en metros).

\begin{tabular}{crrr}
\hline & X & \multicolumn{1}{c}{ Y } & \multicolumn{2}{c}{ Área } \\
\hline B1 & 3 & 1 & 3 \\
B2 & 3 & 2 & 6 \\
CENTROIDES & 3 & 1,7 & 5 \\
\hline
\end{tabular}

Fuente: Los autores. 
Tabla 10. Nueva matriz de recorrido (en metros).

\begin{tabular}{rrrrrrr}
\hline & A & B & C & D & E & F \\
\hline A & & 5 & 3 & 7 & 6 & 3,5 \\
B & 5 & & 2 & 3 & 1,5 & 6 \\
C & 3 & 2 & & 5,5 & 4,5 & 4 \\
D & 7 & 3 & 5,5 & & 2,5 & 7,5 \\
E & 6 & 1,5 & 4,5 & 2,5 & & 6,5 \\
F & 3,5 & 6 & 4 & 7,5 & 6,5 & \\
\hline
\end{tabular}

Fuente: Los autores.

Tabla 11. Nueva matriz de costos (en pesos).

\begin{tabular}{rrrrrrr}
\hline & A & B & C & D & E & F \\
\hline A & 0 & 32469 & 58444 & 0 & 194814 & 0 \\
B & 32469 & 0 & 155851 & 214296 & 0 & 428591 \\
C & 58444 & 155851 & 0 & 0 & 0 & 285727 \\
D & 0 & 214296 & 0 & 0 & 0 & 0 \\
E & 194814 & 0 & 146111 & 0 & 0 & 0 \\
F & 0 & 428591 & 285727 & 0 & 0 & 0 \\
\hline & & & & Total \$ & $\mathbf{2 . 8 8 6 . 4 9 6}$ \\
\hline
\end{tabular}

Fuente: Los autores.

Tabla 12. Matriz de intercambios B-E (en pesos).

\begin{tabular}{ccccccc}
\hline & A & B & C & D & E & F \\
\hline A & 0 & 38963 & 58444 & 0 & 162345 & 0 \\
B & 38963 & 0 & 350665 & 178580 & 0 & 464307 \\
C & 58444 & 350665 & 0 & 0 & 0 & 285727 \\
D & 0 & 178580 & 0 & 0 & 0 & 0 \\
E & 162345 & 0 & 64938 & 0 & 0 & 0 \\
F & 0 & 464307 & 285727 & 0 & 0 & 0 \\
\hline \multicolumn{7}{c}{ Total } \\
\hline
\end{tabular}

Fuente: Los autores.

Tabla 13. Matriz de intercambios B-D (en pesos).

\begin{tabular}{rrrrrrr}
\hline & A & B & C & D & E & F \\
\hline A & 0 & 45457 & 58444 & 0 & 194814 & 0 \\
B & 45457 & 0 & 428591 & 250012 & 0 & 535739 \\
C & 97407 & 272740 & 0 & 0 & 0 & 285727 \\
D & 0 & 250012 & 0 & 0 & 0 & 0 \\
E & 194814 & 0 & 146111 & 0 & 0 & 0 \\
F & 0 & 535739 & 285727 & 0 & 0 & 0 \\
\hline & & & & Total \$ & $\mathbf{3 . 6 2 6 . 7 9 1}$ \\
\hline
\end{tabular}

Fuente: Los autores.

Tabla 14. Matriz de intercambios E-D (en pesos).

\begin{tabular}{rrrrrrr}
\hline & A & B & C & D & E & F \\
\hline A & 0 & 32469 & 58444 & 0 & 227283 & 0 \\
B & 32469 & 0 & 155851 & 107148 & 0 & 428591 \\
C & 116888 & 116888 & 0 & 0 & 0 & 285727 \\
D & 0 & 107148 & 0 & 0 & 0 & 0 \\
E & 227283 & 0 & 178580 & 0 & 0 & 0 \\
F & 0 & 428591 & 285727 & 0 & 0 & 0 \\
\hline \multicolumn{7}{c}{ Total } \\
\hline
\end{tabular}

Fuente: Los autores.

\subsection{Análisis de costos}

Costo actual, distribución versus costo de la nueva distribución.

En la Tabla 15 y Tabla 16 se muestra la matriz de la distribución inicial en donde se obtuvo un costo de \$3.074.817 y la matriz de la distribución después de haber aplicado el método CRAFT, la cual dio un costo de \$2.636.485. Se puede notar cómo al reubicar los departamentos C y B se disminuye el costo de transporte. Las casillas seleccionadas con color amarillo muestran exactamente en cuál de los departamentos se disminuyó los costos de transporte.

Después de haber determinado el costo de cada una de las distribuciones, se obtuvo que al implementar la nueva propuesta se disminuye su costo en un $7 \%$ que corresponde a $\$ 438.322$. (ver Figura 9)

\section{Conclusiones}

Los diferentes análisis realizados del proceso de producción de pintura elaborado en el Laboratorio de Materiales Compuestos de la Escuela Naval de Suboficiales ARC "Barranquilla", nos permitió determinar las condiciones y medios con los cuales es manipulada la materia prima en las diferentes partes del proceso, para posteriormente, realizar una evaluación de qué tan adecuados son los equipos destinados para el manejo del material.

Dicha evaluación nos permitió realizar las siguientes modificaciones a los instrumentos utilizados para la elaboración de la pintura vinilo tipo I, elaborada en la escuela naval de suboficiales ARC "Barranquilla":

- Al recipiente donde se mide el agua (envase de cuñete), se le señalaron las diferentes medidas con el fin de tener una mejor precisión a la hora de pesar el agua, evitando así reproceso por no tener la medida exacta.

- En cuanto al instrumento con el que se pesa la materia prima, se cambió por vasos de precipitados, ayudando a tener medidas más precisas, evitando que se agregue más de lo necesario a la maquina mezcladora. 


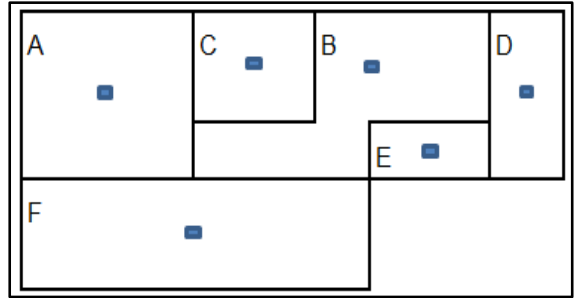

Figura 5. Distribución del laboratorio después de la primera interacción.

Fuente: Los autores.

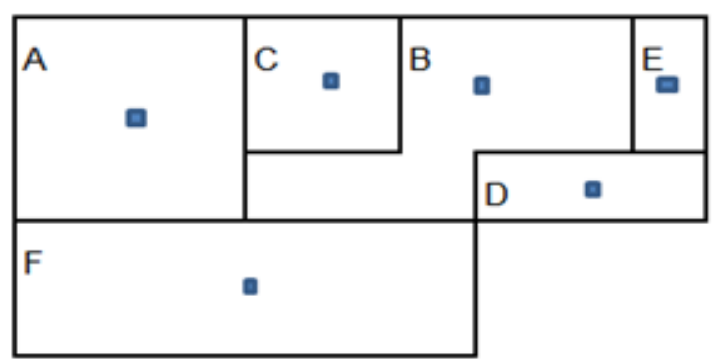

Figura 6. Distribución del laboratorio después de la segunda interacción.

Fuente: Los autores.

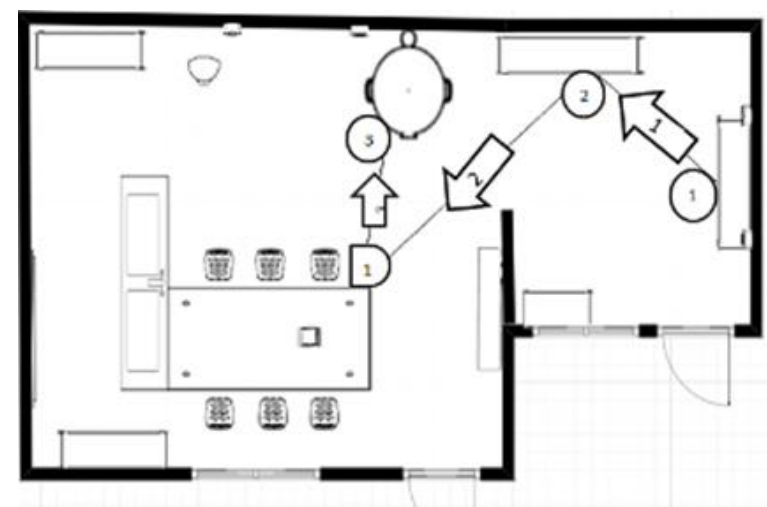

Figura 7. Nueva distribución método de CRAFT.

Fuente: Los autores.
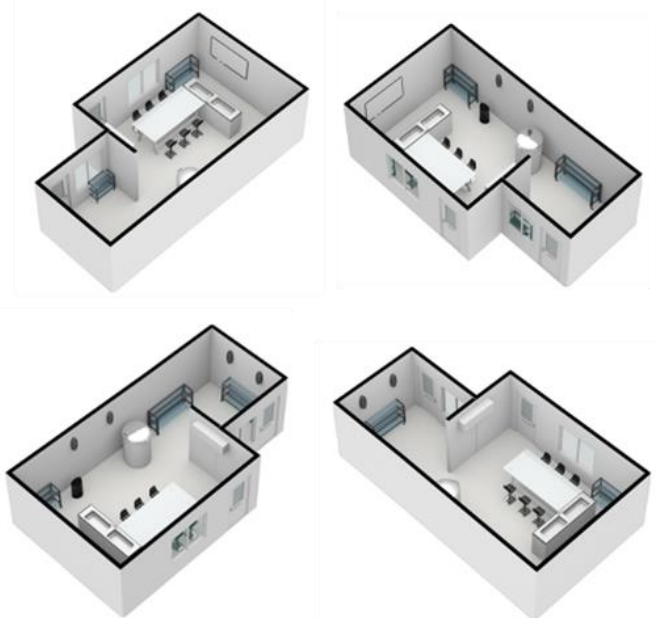

Figura 8. Nueva distribución método de CRAFT. Fuente: Los autores.
Tabla 15. Costo distribución actual (en pesos).

\begin{tabular}{rrrrrrr}
\hline & A & B & C & D & E & F \\
\hline A & 0 & 19481 & 107148 & 0 & 227283 & 0 \\
B & 19481 & 0 & 194814 & 285727 & 0 & 250012 \\
& & & & & & \\
C & 107148 & 194814 & 0 & 0 & 0 & 428591 \\
D & 0 & 285727 & 0 & 0 & 0 & 0 \\
E & 227283 & 0 & 48704 & 0 & 0 & 0 \\
F & 0 & 250012 & 428591 & 0 & 0 & 0 \\
\hline \multicolumn{7}{c}{ Total } \\
\hline
\end{tabular}

Fuente: Los autores.

Tabla 16. Costo distribución propuesta (en pesos).

\begin{tabular}{|c|c|c|c|c|c|c|}
\hline & $\mathbf{A}$ & B & C & D & $\mathbf{E}$ & $\mathbf{F}$ \\
\hline $\mathbf{A}$ & 0 & 35716 & 58444 & 0 & 194814 & 0 \\
\hline B & 35716 & 0 & 194814 & 107148 & 0 & 428591 \\
\hline C & 58444 & 194814 & 0 & 0 & 0 & 250012 \\
\hline D & 0 & 107148 & 0 & 0 & 0 & 0 \\
\hline $\mathbf{E}$ & 194814 & 0 & 97407 & 0 & 0 & 0 \\
\hline $\mathbf{F}$ & 0 & 428591 & 250012 & 0 & 0 & 0 \\
\hline \multicolumn{4}{|c|}{ Total } & \multicolumn{3}{|c|}{2.636 .485} \\
\hline
\end{tabular}

Fuente: Los autores.

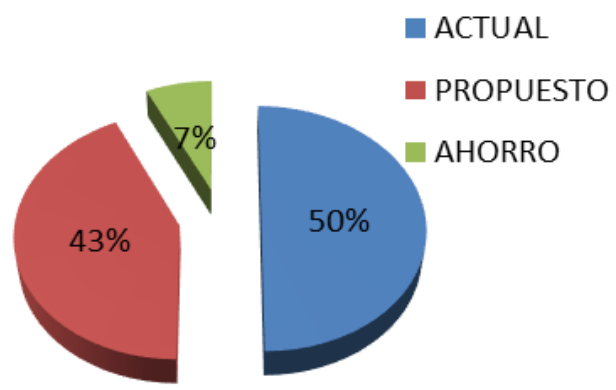

Figura 9. Comparación de costos de la distribución. Fuente: Los autores.

- Para la máquina mezcladora se sugiere implementar un sistema que permita levantar el tanque de mezclado y así, a través de una llave sea envasado el producto final, evitando la contaminación y el desperdicio de la pintura.

Con la implementación de cada uno de los puntos anteriores se logró optimizar el proceso.

Ha quedado establecido que la distribución en planta implica la ordenación de espacios necesarios para movimiento de material, almacenamiento, equipos o líneas de producción, equipos industriales, administración, servicios para el personal y espacios comunes dentro de una instalación productiva propuesta o ya existente, 
buscando aumentar la producción y minimizar costos.

La distribución del laboratorio de materiales compuesto no era la adecuada, los espacios destinados para cada una de las áreas de trabajo no permitían una buena movilidad del operario a cargo, también se realizaban recorridos largos que afectaban el tiempo final de proceso, es por eso por lo que a través del método heurístico CRAFT y del software IIE MICRO-CRAFT, se logró determinar una distribución que disminuyó los costos de transporte del proceso en un 7\%. También se redujo el riesgo de enfermedades profesionales y de accidentes de trabajo, eliminando lugares inseguros, pasos peligrosos y materiales de pasillos. Se obtuvo un menor número de retrasos, reduciéndose los tiempos de espera, al equilibrar los tiempos de trabajo y se disminuyeron las distancias de recorrido.

\section{Referencias}

López, A. (2017). Distribución en planta. Retrieved April 15, 2018, from http://slideplayer.es/slide/11792893/

Muther, R. (1970). Distribución en planta (2 edición). Barcelona-España: McGraw Hill. Retrieved from http://hpcinc.com/wpcontent/uploads/2016/07/Spanish-PPL.pdf

Nahmias, S. (2007). Análisis de la producción y las operaciones (5th ed.). Mexico. Retrieved from https://docs.google.com/viewer? $a=v \&$ pid $=$ sites \&srcid $=Z G V m Y X V s d G R v b W F p b n x w b 3 J 0 Y W Z v$ bGlvdmlydHVhbHBjb2xlaXNhY3VuYXxneDoz NGMwMGJiOTMzMTMzNmM

Platas, J., \& Cervantes, M. (2014). Planeación, diseño y layout de instalaciones (1st ed.). Mexico. Retrieved from https://books.google.com.co/books?id=6jnABgA AQBAJ\&pg $=P A 69 \& l p g=P A 69 \& d q=E l+$ movim iento + del + material $+e s+u n+$ factor + muy + impo rtante + en + la + reducción + de + costos + de + prod ucción, + pues + permite $+q u e+$ los + trabajadores + se + especialicen + en + las + operaciones $+y++n$ o+en+e\#v=onepage
Coronado, J., \& Mejía, G. (2007). Métodos para la distribución en planta. Retrieved from https://sites.google.com/site/jaracohe/home/doc encia/ingenieriademetodos 